ISS: 2602-8085

www.cienciadigital.org

Vol. 3, N³.3, p. 187 - 195, septiembre, 2019

\title{
Índice de masa corporal como factor de riesgo en estudiantes de la carrera de enfermería
}

\section{Body mass index as a risk factor in nursing students}

Wilson Gonzalo Paredes Garcés. ${ }^{1}$, Mery Rocío Rea Guamán. ${ }^{2}$, Janine Maribel Taco Vega. ${ }^{3}$, Silvana Ximena López Paredes. ${ }^{4}$

\section{Recibido: 02-06-2019 / Revisado: 26-06-2019 /Aceptado: 03-08-2019/ Publicado: 06-09-2019}

Abstract DOI: $\underline{\text { https://doi.org/10.33262/cienciadigital.v3i3.3.792 }}$

The body mass index of students varies according to the lifestyle, intervening factors such as; disorders in nutritional habits, excess of school activities, sleep, rest, change of geographical area, and other patterns. Objective: To determine the body mass index as a risk factor in nursing students. Methodology: Population 651 students measured in weight and height and from these in the SPSS their nutritional status is determined, according to WHO. Results: BMI 52 students (11.1\%) malnourished, $253(54.1 \%)$ normal state, $124(26.5 \%)$ overweight, 23 (4.9\%) obesity and 4 (0.9\%) morbid obesity. Discussion the BMI indicates risk factors in young people regardless of gender (Parra et al., 2015). Therefore, by obtaining the BMI, the relationship could be evidenced as a risk factor for health. Conclusion: A high or low BMI constitutes a risk to health, due to lack, excess or bad eating habits.

Keywords: Weights and body measurements, physical appearance, anthropometry, body mass index.

\section{RESUMEN}

El índice de masa corporal de estudiantes varía según el estilo de vida, interviniendo factores como; trastornos en hábitos nutricionales, exceso de actividades escolares, sueño, descanso, cambio de zona geográfica, y otros patrones. Objetivo: Determinar

\footnotetext{
${ }^{1}$ UEB Universidad de Bolívar, Ciencias de la Salud Enfermería, gparedes112004@ @otmail.com

${ }^{2}$ UEB Universidad de Bolívar, Ciencias de la Salud Enfermería, rea_mery@ hotmail.com

${ }^{3}$ UEB Universidad de Bolívar, Ciencias de la Salud Enfermería, janytave@yahoo.es

${ }^{4}$ UEB Universidad de Bolívar, Ciencias de la Salud Enfermería, silvhana@ hotmail.es
} 
el índice de masa corporal como factor de riesgo en los estudiantes de Enfermería. Metodología: Población 651 estudiantes medidos en peso y talla y a partir de éstos en el SPSS se determina su estado nutricional, según OMS. Resultados: IMC 52 estudiantes (11,1\%) desnutridos, 253 (54,1\%) estado normal, 124 (26,5\%) sobrepeso, $23(4,9 \%)$ obesidad y 4 (0,9\%) obesidad mórbida. Discusión El IMC indica factores de riesgo en jóvenes independientemente del sexo, por lo cual mediante la obtención del IMC se podría evidenciar la relación como un factor indicador de riesgo a la salud. Conclusión: Un IMC elevado o bajo constituye riesgo para la salud, por carencia, exceso o malos hábitos alimenticios.

Palabras Clave: Pesas y medidas corporales, apariencia física, antropometría, Índice de masa corporal.

\section{INTRODUCCIÓN:}

\section{Antecedentes}

El IMC que en adelante se identificará como índice de masa corporal responde a una fórmula matemática que relaciona la estatura y el peso de cada persona, mide el porcentaje de grasa corporal de cada individuo (htt2).Se utiliza para evaluar la malnutrición y la obesidad en niños adolescentes y adultos(Carrascosa et al., 2018) Ecuador tiene alta proporción de adolescentes con retardo en crecimiento en las provincias de Bolívar con (41.0\%), mientras que la prevalencia de sobrepeso tiene Azuay (27\%), El Oro (24\%) y Esmeraldas (24.3\%); en cambio Bolívar muestra prevalencia más baja de sobrepeso con $8.9 \%$ en adolescentes. (Wilma Freire). Este dato incrementa al 62,8 \% en adultos (de 19 a 59 años). (Encalada). (INEC), determinar el IMC a partir de un análisis estadístico en relación con los rangos nutricionales normales establecidos por la Organización Mundial de la Salud. Resultados aproximadamente $17 \%$ de estudiantes se encuentran fuera de rangos normales, lo cual amerita realizar una intervención que permita mejorar estos estándares y con ello prever mejor rendimiento y salud en general. Metodología Se trabajó con la totalidad de la población 712 hombres y mujeres, de los cuales 58 se perdieron por inasistencias o retiros, se utilizaron un altímetro y una báscula mecánica, que se calibraron similarmente. El SPSS 23 permitió el procesamiento estadístico a partir de peso y talla, El estado nutricional se 
clasifico en; desnutrido, normal, sobrepeso, obesidad, y obesidad mórbida tomados de la OMS para la determinación del estado nutricional. Discusión Datos de referencia del IMC permitieron determinar el estado nutricional de una persona de manera exacta. (Organización de Mundial de la salud, n.d.)

El IMC se correlaciona con el porcentaje de grasa corporal, en particular cuando es $\geq 35$ $\mathrm{kg} / \mathrm{m}^{2}$ El IMC se puede calcular fácilmente y sin coste alguno, y está bien correlacionado con diversas causas de morbimortalidad(Gallego Gómez, Hita Contreras, Lomas-Vega, \& Martínez-Amat, 2011).

El periodo universitario en el adulto joven, y su autonomía en la toma de decisiones en el establecimiento de conductas y hábitos, es un momento decisivo para detectar posibles desequilibrios y tomar medidas de corrección(Castelao-Naval et al., 2018).

A partir del cumplimiento del objetivo se determina con la evaluación del IMC que el estilo de vida del universitario varía ante factores como; el abandono del hogar, familia, incremento de actividades escolares, sueño, tiempo de descanso, cambio de zona geográfica e incluso su adaptación, aspectos que invitan a ser investigados.

Aquello que parece una virtud en Bolívar con bajo sobre peso es más bien una consecuencia de los promedios del bajo peso en su población.

\section{METODOLOGÍA}

Enfoque Cuali cuantitativo descriptivo y corte transversal, esto permitió conocer las condiciones nutricionales de los estudiantes de la Escuela de Enfermería de la UEB, a través del peso y talla que se obtuvo en la medición antropométrica. La recolección de datos se realiza con la modalidad de censo y comprendió 712 estudiantes universitarios de Enfermería. 58 datos estudiantiles fueron perdidos (inasistencia a toma de referencia, retiros de la institución o situaciones familiares, económicas, la cual imposibilitó la terminación de la carrera). Por tanto 654 estudiantes (hombres y mujeres) fueron participantes. La unidad de análisis fue obtenida de cada alumno, utilizando la fórmula (talla/peso ${ }^{2}$ ) se obtuvo rangos $<18.5$ y $>30$ con lo cual se estableció el estado nutricional creando una variable denominada (nutricional) a la cual se le asignaron; rangos inferiores, intermedios y superiores, correspondientes a desnutridos, normal, sobrepeso, obesidad mórbida valores de categoría ordinal. Los Instrumentos utilizados fueron, el altímetro y una báscula mecánica, que se 
calibraron de manera previa al día de cada medición. Las medidas de peso y talla fueron registradas de curso en curso por los estudiantes investigadores, a toda la Escuela de Enfermería. El IMC $\left(\mathrm{kg} / \mathrm{m}^{2}\right)$ y el estado nutricional se calcularon para cada medición antropométrica. Con el programa estadístico SPSS 23 se calculó una desviación estándar de 0,77, media de 2,30, máximo de 5,00 y un mínimo de 1,00 basados en los datos de IMC.

Una vez ingresadas, establecidas y calculadas las variables de peso, talla, IMC y estado nutricional se procedieron al análisis de las tablas de frecuencia los resultados de los datos ingresados de acuerdo al requerimiento.

Hi 48,9\% son parte de un de factor de riesgo ya sea por bajo o sobrepeso acorde con las medidas obtenidas.

Figura 1. Estado nutricional y el índice de masa corporal

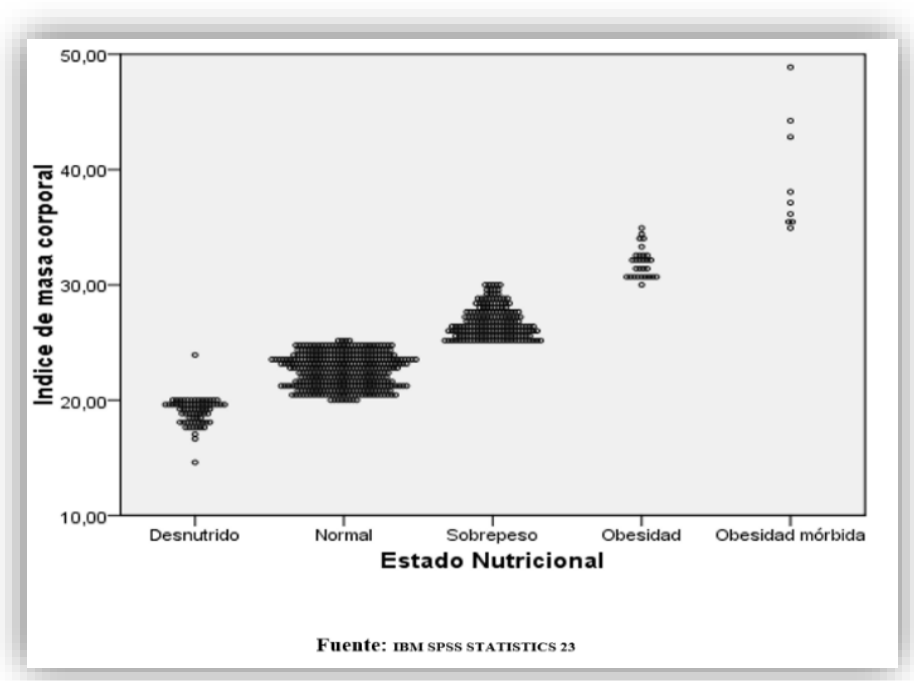

Fuente: Base de datos IMC (programa Estadístico SPSS)

\section{RESULTADOS}

Mediante el IMC de cada estudiante, se pudo determinar su estado nutricional determinándolo según valores referenciales de la OMS (<18,5 "desnutrido bajo peso", valores entre 18,5 a 24,9 "normal" entre 25 y 29,9 "sobrepeso" 30 obesidad >30 "obesidad mórbida". Determinando así en la población universitaria la frecuencia y porcentaje de los casos de; desnutrición, normal, sobrepeso, obesidad y obesidad mórbida. 
De acuerdo con las frecuencias y porcentajes de la población total, 70 son las personas que presentan los casos de desnutrición que representan el 9,8 \% 364 estudiantes con un porcentaje del 51,1\% representan los casos normales, 182 estudiantes con un porcentaje de 25,6\% son casos de sobrepeso, 29 estudiantes con un porcentaje de 4,1\% representan a los casos de obesidad y 9 estudiantes con un porcentaje de 1,3\% son casos de obesidad mórbida (Tabla 1).

Tabla 1. Estado Nutricional

\begin{tabular}{|c|c|c|c|c|c|}
\hline \multicolumn{5}{|c|}{} & \multicolumn{2}{c}{ Estado Nutricional } \\
\hline & Frecuencia & Porcentaje & $\begin{array}{c}\text { Porcentaje } \\
\text { válido }\end{array}$ & $\begin{array}{c}\text { Porcentaje } \\
\text { acumulado }\end{array}$ \\
\cline { 2 - 6 } & Desnutrido & 70 & 9,8 & 10,7 & 10,7 \\
\cline { 2 - 6 } & Normal & 364 & 51,1 & 55,7 & 66,4 \\
\cline { 2 - 6 } Válido & Sobrepeso & 182 & 25,6 & 27,8 & 94,2 \\
\cline { 2 - 6 } & Obesidad & 29 & 4,1 & 4,4 & 98,6 \\
\cline { 2 - 6 } & Obesidad mórbida & 9 & 1,3 & 1,4 & 100,0 \\
\cline { 2 - 6 } & Total & 654 & 91,9 & 100,0 & \\
\hline \multirow{2}{*}{ Perdidos } & Sistema & 58 & 8,1 & & \\
\hline & Total & 712 & 100,0 & & \\
\hline
\end{tabular}

Fuente: Base de datos IMC (programa Estadístico SPSS)

\section{Índice de masa corporal}

El IMC corporal ayudó a identificar los casos de desnutrición, sobrepeso y obesidad que son los indicadores más importantes a la hora de determinar el tipo de riesgo que conlleva con la salud y sus diferentes problemas nutricionales. Con lo cual el estado nutricional dado según los valores referencias ayudan a una intervención rápida y oportuna mencionando al IMC como un factor de riesgo ante un exceso o déficit de los valores normales.

Base de datos IMC (programa Estadístico SPSS) 


\section{DISCUSIÓN}

En las sociedades contemporáneas la mal nutrición por déficit o exceso se ha convertido en un problema de salud global. Sin embargo en un análisis general en 651 estudiantes considerando el peso y la talla se obtiene (IMC) ideado por Adolphe Quetelet; por eso conocido también como índice de Quetelet, cuyo resultado va cambiando con la edad, las mediciones obtenidas en el presente trabajo indican que los estudiantes universitarios de la Universidad Estatal de Bolívar presentan un IMC por debajo del nivel de sobrepeso (IMC<25). Resultados similares se han encontrado en estudios con universitarios de otra ciudad como Milagro UNEMI. Estos resultados difieren cuando los observamos desde la perspectiva de sexo, las mujeres presentan un mayor IMC que los hombres.

En el análisis de presente estudio se obtiene que el IMC 52 estudiantes $(11,1 \%)$ están desnutridos, $253(54,1 \%)$ presentan un estado normal, 124 (26,5\%) sobrepeso, 23 (4,9\%) obesidad y $4(0,9 \%)$ obesidad mórbida, relacionando estos resultados con la Encuesta Nacional de Salud y Nutrición (2013), en el Ecuador el 26\% de la población de 12 - 19 años presenta obesidad y en el $63 \%$ de adultos prevalece el sobrepeso. Con relación al sedentarismo el $30 \%$ y $40 \%$ de los hombres y mujeres adultos, respectivamente, no realizan actividades físicas

En contraposición se puede argumentar que el Índice de masa corporal genera la relación entre peso y estatura, pero sin discernir que dicha relación puede generar un falso positivo lejano a la realidad, pudiendo determinar sobrepeso cuando no lo hay, lo cual se debe a que: El IMC no distingue entre estos dos tipos de tejido corporal ejemplo en aquellos con mayor masa corporal como son los atletas sin distinguir musculatura de grasa. La obesidad es una pandemia que afecta todos los niveles socioeconómicos y ya no es un problema único de las mujeres. En estudios realizados con alumnos universitarios de Latinoamérica se encontró que el sobrepeso u obesidad presentaba mayores prevalencias en los factores de riesgo para tener un síndrome metabólico. (Corral) Acorde a la cita se investigaron 13601personas y determinó que el $21 \%$ de hombres y $31 \%$ de las mujeres. Tenían sobrepeso, pese a esto utilizando el porcentaje de grasa corporal se determinaron cambios y ahora 50\% de varones y $62 \%$ del sexo femenino tenían obesidad 
ISSN: 2602-8085

www.cienciadigital.org

Vol. 3, N³.3, p. 187 - 195, septiembre, 2019

Un estudio publicado por el Journal of the American Medical Association en 2005 (Flegal

$\mathrm{KM}$ ) encontró similitud en el riesgo de morir entre personas con sobrepeso y normales.

Un estudio de 2010 que siguieron a 11.000 sujetos durante 8 años concluyó que el IMC no es una buena medida para considerar el riesgo de ataque al corazón, infarto de miocardio o muerte.

UNEMI en el $201437 \%$ de la muestra tiene obesidad y sobrepeso, con prevalencia en las mujeres

Se pudo identificar que el IMC es un factor de riesgo para estudiantes independientemente del sexo, Un IMC elevado o bajo constituye riesgo para la salud, por carencia, exceso o malos hábitos alimenticios; por tal motivo limita su rendimiento académico, es por ello que se recomienda para futuros estudios implementar educación alimentaria nutricional para mejorar la salud de los estudiantes.

\section{Conclusiones}

Con la ayuda del SPSS se estableció una matriz de datos con las características de IMC de estudiantes de enfermería UEB 2018

El presente estudio sirvió para organizar prevención en salud general

Si bien La Provincia de Bolívar aparentemente cuenta con bajos índices de obesidad se puede concluir que más bien responde a los bajos promedios de peso que muestran resultados según el INEN 2010

\section{Bibliografía}

1. Carrascosa, A., Yeste, D., Moreno-Galdó, A., Gussinyé, M., Ferrández, Á., Clemente, M., \& Fernández-Cancio, M. (2018). Índice de masa corporal e índice de masa triponderal de 1.453 niños no obesos ni malnutridos de la generación del milenio. Estudio longitudinal de Barcelona. Anales de Pediatría, 89(3), 137-143. https://doi.org/10.1016/j.anpedi.2017.12.016

2. Castelao-Naval, O., Blanco-Fernández, A., Meseguer-Barros, C. M., ThuissardVasallo, I. J., Cerdá, B., \& Larrosa, M. (2018). Estilo de vida y riesgo de trastorno alimentario atípico en estudiantes universitarios: realidad versus percepción. Enfermería Clínica. https://doi.org/10.1016/j.enfcli.2018.03.002 
3. Gallego Gómez, A. M., Hita Contreras, F., Lomas-Vega, R., \& Martínez-Amat, A. (2011). Estudio comparativo del índice de masa corporal y el equilibrio postural en estudiantes universitarios sanos. Fisioterapia, 33(3), 93-97. https://doi.org/10.1016/j.ft.2011.03.003

4. Lima-Serrano, M., Martínez-Montilla, J. M., Guerra-Martín, M. D., VargasMartínez, A. M., \& Lima-Rodríguez, J. S. (2018). Factores relacionados con la calidad de vida en la adolescencia. Gaceta Sanitaria, 32(1), 68-71. https://doi.org/10.1016/j.gaceta.2016.06.016

5. Organización de Mundial de la salud. (n.d.). Obesidad y sobrepeso. Retrieved October 21, 2018, from

6. Parra, B. E., Manjarrés, L. M., Velásquez, C. M., Agudelo, G. M., Estrada, A., Uscátegui, R. M., ... Parra, OM. V. (2015). Perfil lipídico y consumo de frutas y verduras en un grupo de jóvenes de 10 a 19 años, según el índice de masa corporal. Revista Colombiana de Cardiología, 22(2), 72-80. https://doi.org/10.1016/j.rccar.2014.10.00

7. s.f. <https://es.wikipedia.org/wiki/\%C3\%8Dndice_de_masa_corporal>.

8. Encalada, Valentina. «Sobrepeso en Ecuador, en la mira de la Organización Mundial de la Salud.» 12 de Octubre de 2017.

9. Salud, organización mundial de la salud. OCTUBRE de 2017. <http://www.who.int/mediacentre/factsheets/fs311/es/>.

10. Wilma Freire, Ma. Jose Ramirez. ENSANUT-ECU 2012. QUITO, 2014.

11. World Health Organization.Influenza(seasonal).March2014.Factsheet ${ }^{2} 211$ [consultado10Abr2015] .Disponibleen:http://www.who.int/mediacentre/factsheets/fs211/en/index.htm

12. Martín, V., Castilla, J., Godoy, P., Delgado-Rodríguez, M., Soldevila, N., Fernández-Villa, T., ... Domínguez, Á. (2016). Índice de masa corporal elevado como factor de riesgo de hospitalización por gripe: estudio de casos y controles. Archivos de Bronconeumología, 52(6), 299-307. doi:10.1016/j.arbres.2015.11.006

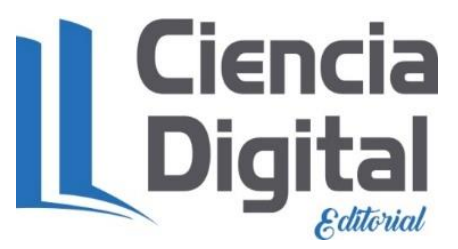




\section{PARA CITAR EL ARTÍCULO INDEXADO.}

Paredes Garcés, W., Rea Guamán, M., Taco Vega, J., \& López Paredes, S. (2019). Índice de masa corporal como factor de riesgo en estudiantes de la carrera de enfermería. Ciencia Digital, 3(3.3), 187-195. https://doi.org/10.33262/cienciadigital.v3i3.3.792

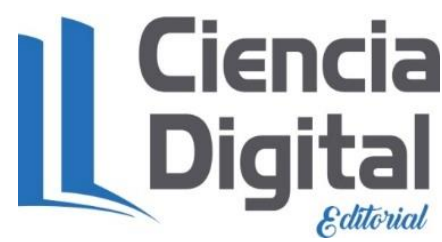

El artículo que se publica es de exclusiva responsabilidad de los autores y no necesariamente reflejan el pensamiento de la Revista Ciencia Digital.

El artículo queda en propiedad de la revista y, por tanto, su publicación parcial y/o total en otro medio tiene que ser autorizado por el director de la Revista Ciencia Digital.
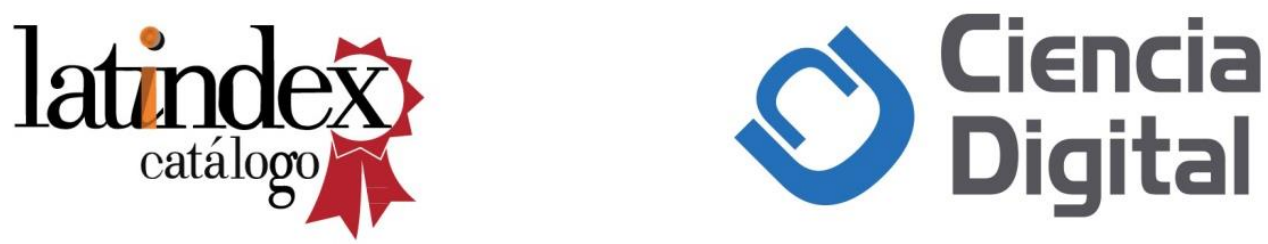